Internat. J. Math. \& Math. Sci.

Vol. 23, No. 6 (2000) 393-398

S0161171200001678

(C) Hindawi Publishing Corp.

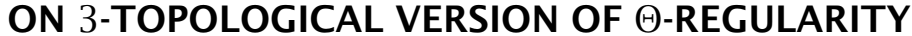

\author{
MARTIN M. KOVÁR
}

(Received 14 July 1998)

\begin{abstract}
We modify the concept of $\theta$-regularity for spaces with 2 and 3 topologies. The new, more general property is fully preserved by sums and products. Using some bitopological reductions of this property, Michael's theorem for several variants of bitopological paracompactness is proved.
\end{abstract}

Keywords and phrases. Spaces with 2 and 3 topologies, $\theta$-regularity, paracompactness.

2000 Mathematics Subject Classification. Primary 54E55, 54A10; Secondary 54D20, 54A20.

1. Preliminaries. The term space $(X, \tau, \sigma, \rho)$ is referred as a set $X$ with three, generally nonidentical topologies $\tau, \sigma$, and $\rho$. We say that $x \in X$ is a $(\sigma, \rho)$ - $\theta$-cluster point of a filter base $\Phi$ in $X$ if for every $V \in \sigma$ such that $x \in V$ and every $F \in \Phi$ the intersection $F \cap \operatorname{cl}_{\rho} V$ is nonempty. If, for every $V \in \sigma$ with $x \in V$, there is some $F \in \Phi$ with $F \subseteq \operatorname{cl}_{\rho} V$, we say that $\Phi(\sigma, \rho)$ - $\theta$-converges to $x$. Then $x$ is called a $(\sigma, \rho)$ - $\theta$-limit of $\Phi$. If $\Phi$ converges or has a cluster point with respect to the topology $\tau$, we say that $\Phi$ $\tau$-converges or has a $\tau$-cluster point.

A family is called $\sigma$-locally finite if it consists of countably many locally finite subfamilies. (This notion has nothing common with the topology also denoted by $\sigma$.) For a family $\Phi \subseteq 2^{X}$, we denote by $\Phi^{F}$ the family of all finite unions of members of $\Phi$. A family $\Phi$ is called directed if $\Phi^{F}$ is a refinement of $\Phi$.

We say that the space $(X, \tau, \sigma, \rho)$ is $(\tau-\sigma)$ (semi-) paracompact with respect to $\rho$ if every $\tau$-open cover of $X$ has a $\sigma$-open refinement which is $(\sigma-)$ locally finite with respect to the topology $\rho$.

The bitopological space $(X, \tau, \sigma)$ is called RR-pairwise (semi-) paracompact if the space is $(\boldsymbol{\tau}-\boldsymbol{\tau})$ (semi-) paracompact with respect to $\sigma$ and $(\sigma-\sigma)$ (semi-) paracompact with respect to $\tau$. We say that $(X, \tau, \sigma)$ is FHP-pairwise (semi-) paracompact if the space is $(\tau-\sigma)$ (semi-) paracompact with respect to $\sigma$ and $(\sigma-\tau)$ (semi-) paracompact with respect to $\tau$. Finally, $(X, \tau, \sigma)$ is said to be $\delta$-pairwise (semi-) paracompact if the space is $(\tau-(\tau \vee \sigma))$ (semi-) paracompact with respect to $\tau \vee \sigma$ and $(\sigma-(\tau \vee \sigma))$ (semi-) paracompact with respect to $\tau \vee \sigma$ (see [7]).

Recall that the topological space $(X, \tau)$ is called (countably) $\theta$-regular [2] if every (countable) filter base in $(X, \tau)$ with a $\theta$-cluster point has a cluster point.

\section{Main results}

THEOREM 2.1. Let $\tau, \sigma, \rho$ be topologies on $X$. The following statements are equivalent:

(i) For every (countable) $\tau$-open cover $\Omega$ of $X$ and each $x \in X$ there is a $\sigma$-open 
neighborhood $U$ of $x$ such that $\operatorname{cl}_{\rho} U$ can be covered by a finite subfamily of $\Omega$.

(ii) Every (countable) $\tau$-closed filter base $\Phi$ with a $(\sigma, \rho)$ - $\theta$-cluster point has a $\tau$ cluster point.

(iii) Every (countable) filter base $\Phi$ with a $(\sigma, \rho)-\theta$-cluster point has a $\tau$-cluster point.

(iv) For every (countable) filter base $\Phi$ in $X$ with no $\tau$-cluster point and every $x \in X$ there are $U \in \sigma, V \in \rho$, and $F \in \Phi$ such that $x \in U, F \subseteq V$, and $U \cap V=\varnothing$.

Proof. Suppose (i). Let $\Phi$ be a (countable) filter base in $X$ with no $\tau$-cluster point. Then $\Omega=\left\{X \backslash \mathrm{cl}_{\tau} F \mid F \in \Phi\right\}$ is a (countable) $\tau$-open directed cover of $X$. Let $x \in X$. By (i) there is $U \in \sigma$ with $x \in U$ and $\operatorname{cl}_{\rho} U \subseteq X \backslash \mathrm{cl}_{\tau} F$ for some $F \in \Phi$. Denote $V=$ $X \backslash \operatorname{cl}_{\rho} U$. Then $x \in U, F \subseteq V \in \rho$ and $U \cap V=\varnothing$. It follows (iv).

The implications (iv) $\Longrightarrow$ (iii) $\Longrightarrow$ (ii) are clear. Suppose (ii). Take any (countable) $\tau$-open cover $\Omega$ of $X$. Then $\Phi=\left\{X \backslash V \mid V \in \Omega^{F}\right\}$ is a $\tau$-closed filter base in $X$ with no $\tau$-cluster point. Let $x \in X$. It follows from (ii) that $x$ is not a $(\sigma, \rho)-\theta$-cluster point of $\Phi$, so there are some $U \in \sigma$ and $V \in \Omega^{F}$ such that $x \in U$ and $(X \backslash V) \cap \operatorname{cl}_{\rho} U=\varnothing$. Then $\operatorname{cl}_{\rho} U \subseteq V$, which implies (i).

DeFinITION 2.2. Let $\tau, \sigma, \rho$ be topologies on $X$. Then $(X, \tau, \sigma, \rho)$ is said to be (countably) $(\tau, \sigma, \rho)-\theta$-regular, if $X$ satisfies any of the conditions (i)-(iv) of Theorem 2.1.

Note that for $\tau=\sigma=\rho$ we obtain the notion of (countably) $\theta$-regular space. Omitting the condition of countability, we get further criteria of $(\tau, \sigma, \rho)$ - $\theta$-regularity.

THEOREM 2.3. Let $\tau, \sigma, \rho$ be topologies on $X$. The following statements are equivalent:

(i) $X$ is $(\tau, \sigma, \rho)$ - $\theta$-regular.

(ii) Every $(\sigma, \rho)$ - $\theta$-convergent filter base $\Phi$ has a $\tau$-cluster point.

(iii) Every $(\sigma, \rho)$ - $\theta$-convergent ultrafilter in $X$ is $\tau$-convergent.

Proof. The implications (i) $\Longrightarrow$ (ii) $\Longrightarrow$ (iii) are clear. Conversely, suppose (iii) and take a filter base $\Phi$ in $X$ with a $(\sigma, \rho)-\theta$-cluster point $x \in X$. Let $\zeta$ be a $\sigma$-open local base of $x$. Then the family $\Phi^{\prime}=\left\{F \cap \operatorname{cl}_{\rho} V \mid F \in \Phi, V \in \zeta\right\}$ is a filter base finer than $\Phi$ and $(\sigma, \rho)$ - $\theta$-converging to $x$. Denote by $\Gamma$ an ultrafilter finer than $\Phi^{\prime}$. Then $\Phi^{\prime} \subseteq \Gamma$ and hence $\Gamma$ also $(\sigma, \rho)$ - $\theta$-converges to $x$. By (iii), $\Gamma$ is $\tau$-convergent to some $y \in X$. Since $\Gamma$ is finer than $\Phi, y$ is a $\tau$-cluster point of $\Phi$.

Similarly as for $\theta$-regularity, there are numbers of simple examples of $(\tau, \sigma, \rho)$ $\theta$-regular spaces, including various modifications of regularity, compactness, local compactness, or paracompactness and we leave them to the reader. Note, for example, that a space $(\tau-\sigma)$ paracompact with respect to $\rho$ is $(\tau, \rho, \sigma)-\theta$-regular.

REMARK 2.4. One can easily check that $(\tau, \sigma, \rho)-\theta$-regularity is preserved by $\tau$ closed subspaces if we consider the corresponding induced topologies on the subspace. On the other hand, as it is shown in [3], even $F_{\sigma}$-subspace of a compact (nonHausdorff) space need not be countably $\theta$-regular.

For a family $\left\{\left(X_{\iota}, \tau_{\iota}, \sigma_{\iota}, \rho_{\iota}\right) \mid \iota \in I\right\}$ denote by $\tau, \sigma, \rho$ the corresponding sum (product) topologies on $X=\sum_{\iota \in I} X_{l}\left(X=\Pi_{l \in I} X_{l}\right)$. It is an easy exercise to prove that the topological sum $X$ of $\left(\tau_{\iota}, \sigma_{\iota}, \rho_{\iota}\right)-\theta$-regular spaces $X_{\iota}$, where $\iota \in I$, is $(\tau, \sigma, \rho)-\theta$-regular. 
THEOREM 2.5. Let $X=\sum_{\iota \in I} X_{\iota}$ be the sum space for the family $\left\{\left(X_{\iota}, \tau_{\iota}, \sigma_{\iota}, \rho_{\iota}\right) \mid \iota \in I\right\}$ with the corresponding sum topologies $\tau, \sigma, \rho$. Suppose that every $X_{\iota}$ is $\left(\tau_{\iota}, \sigma_{\iota}, \rho_{\iota}\right)-\theta$ regular. Then $X$ is $(\tau, \sigma, \rho)$ - $\theta$-regular.

THEOREM 2.6. Let $X=\Pi_{l \in I} X_{\iota}$ be the product space for the family $\left\{\left(X_{l}, \tau_{l}, \sigma_{l}, \rho_{\iota}\right) \mid\right.$ $\iota \in I\}$ with the corresponding product topologies $\tau, \sigma, \rho$. Suppose that every $X_{\iota}$ is $\left(\tau_{\iota}, \sigma_{\iota}, \rho_{\iota}\right)-\theta$-regular. Then $X$ is $(\tau, \sigma, \rho)-\theta$-regular.

Proof. Let $\Gamma$ be an ultrafilter in $X$ with $(\sigma, \rho)$ - $\theta$-limit $x=\left(x_{\iota}\right)_{\iota \in I} \in X$. Let $\pi_{l}$ : $X \rightarrow X_{\iota}$ be the canonical projection. Then $\pi_{\iota}(\Gamma)$ is an ultrafilter on $X_{\iota}$ which $\left(\sigma_{\iota}, \rho_{\iota}\right)$ $\theta$-converges to $x_{l}$. But $X_{l}$ is $\left(\tau_{l}, \sigma_{l}, \rho_{l}\right)$ - $\theta$-regular. Hence, $\pi_{l}(\Gamma) \tau_{l}$-converges to some $y_{\iota} \in X_{\iota}$, which implies that $\Gamma \tau$-converges to $y=\left(y_{\iota}\right)_{\iota \in I}$. It follows that $X$ is $(\tau, \sigma, \rho)$ $\theta$-regular.

The productivity of $\theta$-regularity proved in [4] by a different technique now follows as a corollary.

DEFINITION 2.7. A bitopological space $(X, \tau, \sigma)$ is said to be $\alpha$-pairwise (countably) $\theta$-regular if $X$ is (countably) $(\tau, \tau, \sigma)$ - $\theta$-regular and (countably) $(\sigma, \sigma, \tau)$ - $\theta$-regular, $\beta$-pairwise (countably) $\theta$-regular if $X$ is (countably) $(\tau, \sigma, \tau)-\theta$-regular and (countably) $(\sigma, \tau, \sigma)$ - $\theta$-regular, $\gamma$-pairwise (countably) $\theta$-regular if $X$ is (countably) $(\tau, \sigma, \sigma)$ $\theta$-regular and (countably) $(\sigma, \tau, \tau)-\theta$-regular and finally, $\delta$-pairwise (countably) $\theta$ regular if $X$ is (countably) ( $\tau \vee \sigma, \sigma, \tau \vee \sigma)-\theta$-regular and (countably) $(\tau \vee \sigma, \tau, \tau \vee \sigma)$ $\theta$-regular.

REMARK 2.8. Using the characterization (i) in Theorem 2.1 and refining the open covers of the space several times, one can easily check that $\beta$ - and $\gamma$-versions of pairwise $\theta$-regularity are equivalent and imply the $\alpha$-version, but not vice versa. Since every pairwise regular space obviously is $\alpha$-pairwise $\theta$-regular, the real line topologized by the intervals $(-\infty, p), p \in \mathbb{R}$ for $\tau$ and $(q, \infty), q \in \mathbb{R}$ for $\sigma$ is a proper counterexample.

REMARK 2.9. Observe that RR-pairwise paracompact and FHP-pairwise paracompact spaces are $\beta$-pairwise $\theta$-regular and it can be easily seen that a $\beta$-pairwise $\theta$ regular space has both topologies $\theta$-regular.

However, for the following bitopological modifications of well-known Michael's theorem [5], only the $\beta$ - and $\delta$-versions of pairwise (countable) $\theta$-regularity will be useful. In the proof of the next theorem, we slightly modify the technique used in [3].

THEOREM 2.10. Let $\sigma_{1}, \sigma_{2}, \sigma_{3}, \sigma_{4}$, be topologies on $X$. Let $X$ be $\left(\sigma_{1}-\sigma_{2}\right)$ semiparacompact with respect to $\sigma_{3},\left(\sigma_{4}-\sigma_{3}\right)$ semiparacompact with respect to $\sigma_{2}$ and countably $\left(\sigma_{2}, \sigma_{4}, \sigma_{2}\right)$ - $\theta$-regular. Then $X$ is $\left(\sigma_{1}-\sigma_{2}\right)$ paracompact with respect to $\sigma_{3}$.

Proof. Let $\Omega$ be a $\sigma_{1}$-open cover of $X$. Since $X$ is $\left(\sigma_{1}-\sigma_{2}\right)$ semiparacompact with respect to $\sigma_{3}$, it follows that $\Omega$ has a $\sigma_{2}$-open refinement, say $\Omega^{\prime}=\bigcup_{i=1}^{\infty} \Omega_{i}$, where every $\Omega_{i}$ is a locally finite with respect to $\sigma_{3}$ family refining $\Omega$.

Let $U_{n}=\bigcup\left\{U \mid U \in \Omega_{i}, i \leq n\right\}$ for every $n \in \mathbb{N}$. The family $\left\{U_{n}\right\}_{n \in \mathbb{N}}$ is a countable $\sigma_{2}$-open increasing cover of $X$ and since $X$ is countably $\left(\sigma_{2}, \sigma_{4}, \sigma_{2}\right)$ - $\theta$-regular, there exists a $\sigma_{4}$-open cover $\Phi$ of $X$ whose $\sigma_{2}$-closures refine $\left\{U_{n}\right\}_{n \in \mathbb{N}}$. Because $X$ is $\left(\sigma_{4}-\sigma_{3}\right)$ 
semiparacompact with respect to $\sigma_{2}$, $\Phi$ has a $\sigma_{3}$-open refinement, say $\Phi^{\prime}=\bigcup_{i=1}^{\infty} \Phi_{i}$, consisting of families $\Phi_{i}$ which are locally finite with respect to $\sigma_{2}$. For every $n \in \mathbb{N}$, let

$$
V_{n}=\bigcup\left\{B \mid B \in \Phi_{i}, \mathrm{cl}_{\sigma_{2}} B \subseteq U_{j}, i+j \leq n\right\} .
$$

The family $\left\{V_{n}\right\}_{n \in \mathbb{N}}$ is a $\sigma_{3}$-open increasing cover of $X$. Because the family $\bigcup_{i=1}^{n} \Phi_{i}$ is locally finite with respect to $\sigma_{2}$, we have $\operatorname{cl}_{\sigma_{2}} V_{n} \subseteq U_{n-1}$. Finally, for every $n \in \mathbb{N}$ and $U \in \Omega_{n}$, let

$$
W_{n}(U)=U \backslash \mathrm{cl}_{\sigma_{2}} V_{n} .
$$

It can be easily seen that the family $\Gamma=\left\{W_{n}(U) \mid n \in \mathbb{N}, U \in \Omega_{n}\right\}$ is a $\sigma_{2}$-open cover of $X$ which is a refinement of $\Omega$ locally finite with respect to $\sigma_{3}$. Indeed, for every $x \in X$ let $k \in \mathbb{N}$ be the least index such that $x \in U$ for some $U \in \Omega_{k}$. Since $\operatorname{cl}_{\sigma_{2}} V_{k} \subseteq U_{k-1}$, it follows that $x \in W_{k}(U)$. Hence $\Gamma$ is a $\sigma_{2}$-open cover which, obviously, refines $\Omega$. To see that $\Gamma$ is locally finite with respect to $\sigma_{3}$, let $x \in X$ and let $m \in \mathbb{N}$ be any index such that $x \in V_{m}$. Because $\left\{V_{n}\right\}_{n \in \mathbb{N}}$ is an increasing family, we have $V_{m} \cap W_{n}(U)=\varnothing$ for every $n \geq m, U \in \Omega_{n}$.

But the family $\bigcup_{i=1}^{m} \Omega_{i}$ is locally finite with respect to $\sigma_{3}$. Let $S$ be a $\sigma_{3}$-neighborhood of $x$, intersecting at most finitely many elements of $\bigcup_{i=1}^{m} \Omega_{i}$. Since for every $i=$ $1,2, \ldots, m, U \in \Omega_{i}$, we have $W_{i}(U) \subseteq U$, the set $S \cap V_{m}$ is a $\sigma_{3}$-neighborhood of $x$, meeting only finitely many sets of the cover $\Gamma$. Hence $\Gamma$ is locally finite with respect to $\sigma_{3}$ and therefore $X$ is $\left(\sigma_{1}-\sigma_{2}\right)$ paracompact with respect to $\sigma_{3}$.

In order to obtain a theorem for a bitopological space $\left(X, \tau_{1}, \tau_{2}\right)$ from Theorem 2.10 it can be easily seen that there are only three meaningful possibilities for identifying the topologies $\sigma_{1}, \sigma_{2}, \sigma_{3}, \sigma_{4}$.

CASE (i). $\tau_{1}=\sigma_{1}=\sigma_{4}$ and $\tau_{2}=\sigma_{2}=\sigma_{3}$.

COROLlaRY 2.11. Let $X$ be countably $\left(\tau_{2}, \tau_{1}, \tau_{2}\right)-\theta$-regular and $\left(\tau_{1}-\tau_{2}\right)$ semiparacompact with respect to $\tau_{2}$. Then $X$ is $\left(\tau_{1}-\tau_{2}\right)$ paracompact with respect to $\tau_{2}$.

COROLLARY 2.12. Let $X$ be a bitopological space. Then $X$ is FHP-pairwise paracompact if and only if $X$ is $\beta$-pairwise countably $\theta$-regular and FHP-pairwise semiparacompact.

Proof. It is sufficient to use the previous corollary twice.

Note that Raghavan and Reilly stated [7, Theorem 3.9] from which it would follow that a pairwise regular $\delta$-pairwise semiparacompact space is $\delta$-pairwise paracompact. Unfortunately, (iv) $\Longrightarrow$ (i) in the proof of this theorem is not correct. The authors used [1, Theorem 1.5, page 162] in the proof. However, the assumptions of the theorem are not completely satisfied. They tried to expand a locally finite cover $\mathscr{V}$ to the open one using a closed cover such that every its element meets only finitely many members of $\mathscr{V}$. However, in general the used closed cover is not locally finite or at least closure preserving. That is not sufficient for the expansion, as the following example shows.

EXAMPLE 2.13. Let $C=\mathbb{N} \times\langle-1,1), B=\mathbb{N} \times(0,1)$, and $A=\mathbb{N} \times\langle-1,0\rangle$. We consider the Euclidean topology on $C$ induced from the real plane and let $X=C \cup\{y \mid$ 
$y$ is a nonconvergent ultra-closed filter in $C, B \in y\}$. Let $S(U)=U \cup\{y \mid y \in X \backslash C$, $U \in y$ \} for any $U \subseteq C$ open in $C$. Of course, $X$ is a subspace of the Wallman compactification $\omega C$ and the sets $S(U)$ constitute a topology base for $X$. Since $C$ is normal, $\omega C$ is Hausdorff and hence $X$ is a $T_{3.5}$ space. Denote $A_{n}=\{n\} \times\langle-1,0\rangle$. The family $\Omega=\left\{S(B), A_{1}, A_{2}, A_{3}, \ldots\right\}$ is a locally finite cover of $X$, which has no open locally finite extension.

Indeed, suppose that there are some open $U_{n}$ with $A_{n} \subseteq U_{n}$ for $n \in \mathbb{N}$. Then every $U_{n}$ must meet $B_{n}=\{n\} \times(0,1)$. Choose $x_{n} \in U_{n} \cap B_{n}$ for each $n \in \mathbb{N}$. Let $F_{n}=$ $\left\{x_{n}, x_{n+1}, \ldots\right\}$. Since the sequence $x_{1}, x_{2}, \ldots$ has no cluster point in $C$, the collection $\Phi=\left\{F_{n} \mid n=1,2, \ldots\right\}$ is a closed filter base in $C$ with no cluster point in $C$. It follows that there is a non-convergent ultra-closed filter, say $y \in \omega C$, finer than $\Phi$. But $F_{1} \subseteq B$ and since $F_{1} \in \Phi \subseteq y, B \in y$. Hence $y \in X$. Let $W$ be any open neighborhood of $y$ in $X$. There is some $V$ open in $C$ with $y \in S(V) \subseteq W$. Then $V \in y$ and hence $V \cap F_{n} \neq \varnothing$ for every $n \in \mathbb{N}$. Thus for any fixed $m \in \mathbb{N}$ there exists $n \geq m$ such that $x_{n} \in V \subseteq S(V) \subseteq W$ and therefore $W$ intersects infinitely many elements of $\left\{U_{n} \mid n=1,2, \ldots\right\}$. Hence $\Omega$ cannot be expanded to an open locally finite cover.

On the other hand, the previous example does not refute Raghavan-Reilly's theorem, which still remains open as a question. With a different modification of the concept of pairwise regularity the theorem is correct.

COROLlary 2.14. Let $X$ be $\delta$-pairwise countably $\theta$-regular. Then $X$ is $\delta$-pairwise paracompact if and only if $X$ is $\delta$-pairwise semiparacompact.

PRoof. Since $X$ is countably $\left(\boldsymbol{\tau}_{1} \vee \tau_{2}, \boldsymbol{\tau}_{1}, \boldsymbol{\tau}_{1} \vee \boldsymbol{T}_{2}\right)-\theta$-regular and $\left(\boldsymbol{\tau}_{1}-\left(\boldsymbol{\tau}_{1} \vee \tau_{2}\right)\right)$ semiparacompact with respect to $\tau_{1} \vee \tau_{2}$, it follows that $X$ is $\left(\tau_{1}-\left(\tau_{1} \vee \tau_{2}\right)\right)$ paracompact with respect to $\tau_{1} \vee \tau_{2}$ by Corollary 2.11. But $X$ is also countably $\left(\tau_{1} \vee \tau_{2}, \tau_{2}, \tau_{1} \vee\right.$ $\left.\boldsymbol{T}_{2}\right)$ - $\theta$-regular and $\left(\boldsymbol{T}_{2}-\left(\boldsymbol{T}_{1} \vee \boldsymbol{T}_{2}\right)\right)$ semiparacompact with respect to $\boldsymbol{\tau}_{1} \vee \boldsymbol{T}_{2}$ which implies, also by Corollary 2.11 , that $X$ is $\left(\tau_{2}-\left(\tau_{1} \vee \tau_{2}\right)\right)$ paracompact with respect to $\tau_{1} \vee \tau_{2}$. Hence $X$ is $\delta$-pairwise paracompact in topologies $\tau_{1}, \tau_{2}$.

REMARK 2.15. Note that the space $X$ constructed in Example 2.13 is $T_{3.5}$ but not normal-the sets $A, X \backslash C$ are closed, pairwise disjoint but they have no disjoint neighborhoods.

CASE (ii). $\boldsymbol{\tau}_{1}=\sigma_{1}=\sigma_{2}$ and $\tau_{2}=\sigma_{3}=\sigma_{4}$.

COROLlarY 2.16. Let $X$ be a bitopological space. Then $X$ is RR-pairwise paracompact if and only if $X$ is $\beta$-pairwise countably $\theta$-regular and RR-pairwise semiparacompact.

CASE (iii). $\tau_{1}=\sigma_{1}=\sigma_{3}$ and $\tau_{2}=\sigma_{2}=\sigma_{4}$.

COROLLARY 2.17. Let $\tau_{1}, \tau_{2}$ be countably $\theta$-regular topologies of X. Suppose that $X$ is $\left(\tau_{1}-\tau_{2}\right)$ semiparacompact with respect to $\tau_{1}$, and $\left(\tau_{2}-\tau_{1}\right)$ semiparacompact with respect to $\tau_{2}$. Then $X$ is $\left(\tau_{1}-\tau_{2}\right)$ paracompact with respect to $\tau_{1}$ and $\left(\tau_{2}-\tau_{1}\right)$ paracompact with respect to $\tau_{2}$.

Finally, remark that modifying properly the concept of $\sum$-space for bitopological spaces, combining Theorem 2.6 and the corollaries of Theorem 2.10 similar results as 
in [4] (see [6, Nagami's theorem]) for the countable product of paracompact $\sum$-spaces without necessity of Hausdorff-type separation are also possible.

ACKNOWLEDGEMENT. This research is supported by grant GA ČR 201/97/0216.

\section{REFERENCES}

[1] J. Dugundji, Topology, Allyn and Bacon Inc., Boston, Mass., 1978. MR 57\#17581.

[2] D. S. Janković, $\theta$-regular spaces, Internat. J. Math. Math. Sci. 8 (1985), no. 3, 615-619. MR 87h:54030. Zbl 577.54012.

[3] M. M. Kovár, On A-regular spaces, Internat. J. Math. Math. Sci. 17 (1994), no. 4, 687-692. MR 95h:54001. Zbl 809.54017.

[4] _ A remark on $\Theta$-regular spaces, Internat. J. Math. Math. Sci. 21 (1998), no. 1, 199200. CMP 1486 978. Zbl 888.54027.

[5] E. Michael, A note on paracompact spaces, Proc. Amer. Math. Soc. 4 (1953), 831-838. MR 15,144b. Zbl 052.18701.

[6] K. Nagami, 乏-spaces, Fund. Math. 65 (1969), 169-192. MR 41\#2612. Zbl 181.50701.

[7] T. G. Raghavan and I. L. Reilly, A new bitopological paracompactness, J. Austral. Math. Soc. Ser. A 41 (1986), no. 2, 268-274. MR 87h:54057. Zbl 609.54023.

KovÁr: DePartment of MATHematics, Faculty of Electrical ENGineering AND COMPUTER SCIENCE, TECHNICAL UNIVERSITY OF BRNO, TECHNiCKÁ 8, 61669 BRNO, CZECH REPUbliC

E-mail address: kovar@dmat. fee.vutbr.cz 


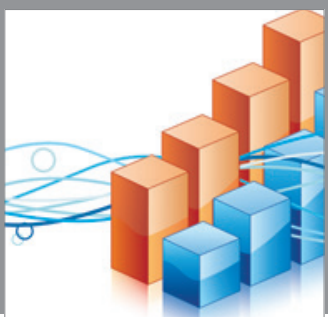

Advances in

Operations Research

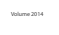

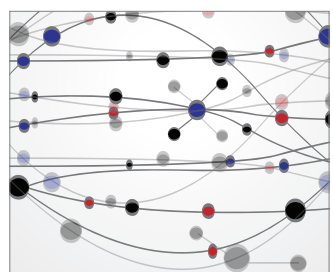

\section{The Scientific} World Journal
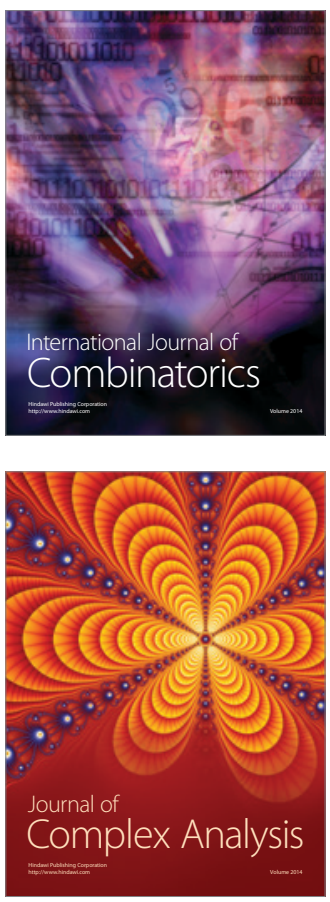

International Journal of

Mathematics and

Mathematical

Sciences
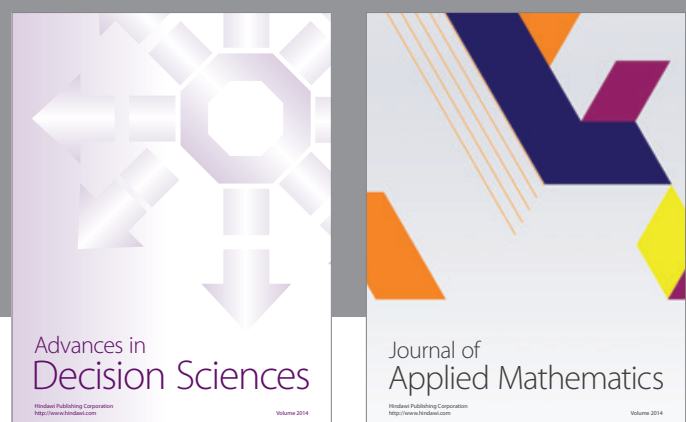

Journal of

Applied Mathematics
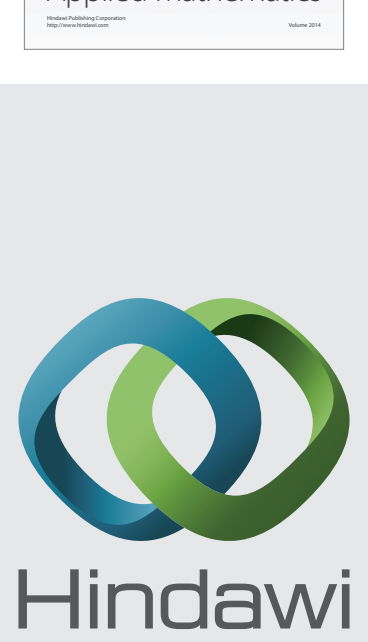

Submit your manuscripts at http://www.hindawi.com
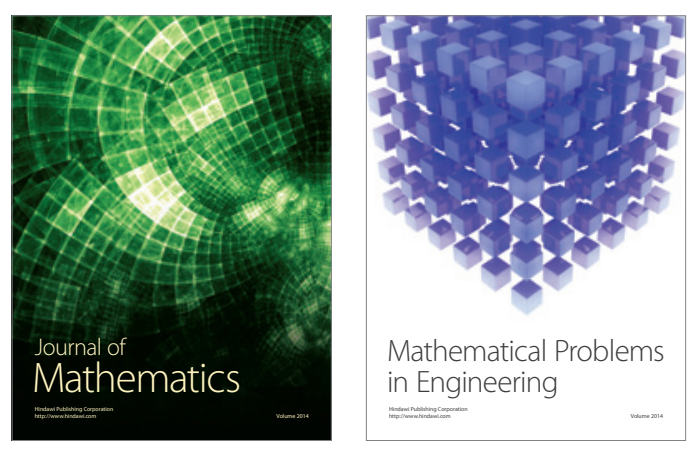

Mathematical Problems in Engineering
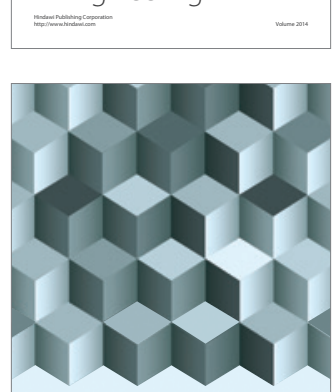

Journal of

Function Spaces
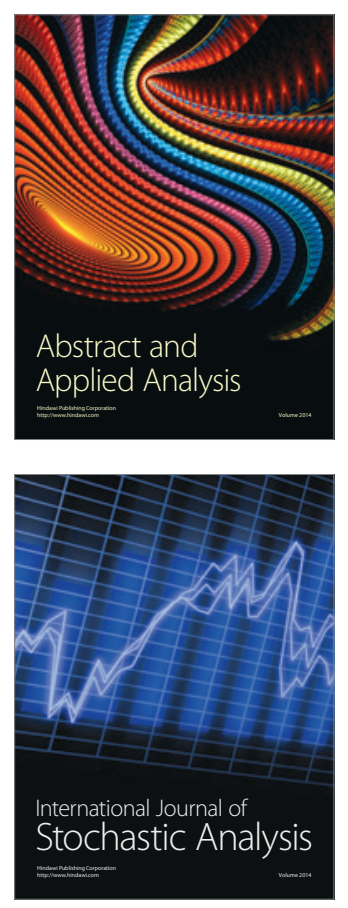

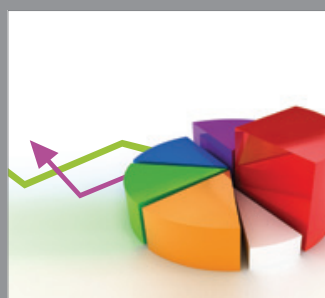

ournal of

Probability and Statistics

Promensencen
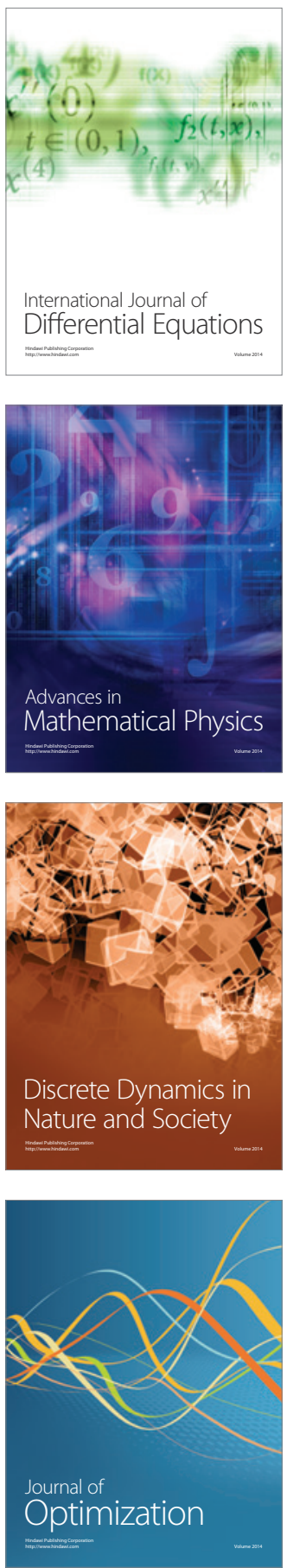